\title{
COMPORTAMENTOS E ATITUDES DOS ESTUDANTES DO ENSINO SUPERIOR EM RELAÇÃO À SEXUALIDADE
}

\author{
Ana Maria Baptista Oliveira Dias Malva Vaz. \\ $\mathrm{RN}$; MS; PhD \\ Instituto Politécnico de Castelo Branco \\ anamariavaz@gmail.com \\ https://doi.org/10.17060/ijodaep.2017.n1.v2.937
}

Fecha de Recepción: 14 Febrero 2017

Fecha de Admisión: 1 Abril 2017

\section{RESUMO}

A sexualidade e a forma como deve ser abordada ainda não tem soluções e os jovens continuam a ter as atitudes/comportamentos que entendem e nem sempre os resultados que daí advêm são os melhores. 0 objetivo é estudar a sexualidade nos jovens estudantes que frequentam $01^{0}$ ano do ensino superior (área da saúde), considerando que na sua maioria se encontram pela primeira vez fora do seu agregado familiar, com vivências próprias dos estudantes que ingressam no ensino superior e também porque é nesta fase da vida que se inicia uma maturação sexual, muito perto da idade adulta.

0 estudo é de natureza quantitativa onde são evidenciadas as atitudes e os comportamentos sexuais dos jovens, que já iniciaram a sua atividade sexual $(64,6 \%)$.

A escala utilizada apresenta quatros dimensões que se subdividem em:

Intenção comportamental: praticar "sexo seguro", que é entendido como uso de preservativo. Reações: sentimentos positivos e negativos do sujeito face à utilização do preservativo e crenças sobre as consequências positivas ou negativas associadas ao desempenho desse comportamento. Norma subjetiva: julgamento subjetivo sobre o grau de aprovação-desaprovação de certas pessoas significativas para o sujeito (pais, amigos, companheiro) sobre 0 uso de preservativo. Percepção de controlo: expectativas de auto-eficácia, julgamento subjetivo sobre o grau de convicção na dificuldade ou facilidade de usar (ou fazer usar) o preservativo no próximo encontro sexual.

Concluímos que existe uma grande percentagem que utiliza preservativo $(36 \%)$, seguindo-se uma dupla proteção, preservativo e pilula $(26,1 \%)$. A maioria dos que tiveram relações sexuais, $(65 \%)$ voltaram a ter relações sexuais com 0 mesmo parceiro.

Os profissionais de saúde devem compreender a complexidade do problema da sexualidade e ter conhecimento da realidade dos estilos de vida adotados pelos jovens, para poderem definir estratégias para a promoção da saúde e intervir nas causas.

Palavras chave: sexualidade; jovens; comportamentos; atitudes; contracepção 


\section{COMPORTAMENTOS E ATITUDES dOS ESTUDANTES DO ENSINO SUPERIOR EM RELAÇÃO À SEXUALIDADE}

\section{ABSTRACT}

Sexuality and the way it should be addressed still has no solutions and the young people continue to have the attitudes/ behaviours they deem appropriate and the results that follow aren't always the best. The objective is to study the sexuality in young students who attend the first year of higher education (area of health), considering that most of them are for the first time out of their family, with typical experiences of students entering higher education, and also because it is at this stage of life that sexual maturation begins, very close to adulthood.

The study is of a quantitative nature, putting in evidence the attitudes and sexual behaviours of young people who have already begun their sexual activity $(64.6 \%)$.

The scale used has four dimensions that are subdivided into:

Behavioural Intent: Practice "safe sex", which is understood as the use of condom. Reactions: positive and negative feelings of the subject towards the use of condom and beliefs about the positive or negative consequences associated with this behaviour. Subjective norm: subjective judgment about the degree of approval-disapproval of certain significant persons to the subject (parents, friends, partner) about the use of condom. Control perception: expectations of self-efficacy, subjective judgment about the degree of conviction in the difficulty or ease of using (or making use) the condom at the next sexual encounter.

We have concluded that there is a large percentage who use the condom (36\%), followed by a double protection, condom and contraceptional pill (26.1\%). Most of those who already had sex, $(65 \%)$ had sex again with the same partner.

Health professionals should understand the complexity of the problem of sexuality and be aware of the reality of the lifestyles adopted by young people, so that they can define strategies for health promotion and intervene in the causes.

Keywords: sexuality; young people; behaviours; attitudes; contraception

\section{INTRODUÇÃO}

A sexualidade deixou de ser algo apenas privado, imbuído de fantasias secretas, e tornou-se um assunto de grande discussão. Nas últimas décadas tem-se verificado uma maior tendência na liberalização dos comportamentos sexuais dos jovens, sendo necessário investir na sua educação sexual.

Considerando que na sua maioria estes jovens se encontram pela primeira vez fora do seu agregado familiar, com vivências próprias dos estudantes que ingressam no ensino superior e também porque é nesta fase da vida que se inicia uma maturação sexual, muito perto da idade adulta. Assim o presente estudo resulta da necessidade de tornar visível a importância de conhecer os comportamentos sexuais dos jovens, os quais estão cada vez mais expostos a comportamentos sexuais de risco, dado que "a adolescência é um período de desenvolvimento cognitivo, emocional e físico frequentemente caracterizado pela existência de oportunidades e vulnerabilidades" (Vilelas, 2009, p.54).

A adolescência constitui-se como um período em que as relações de namoro assumem um contexto de desenvolvimento e de vinculação importante, com implicações para 0 auto-conceito e na auto-estima dos adolescentes (Custódio, 2009).

\section{OBJETIVOS}

Pretende-se com este estudo identificar as atitudes e comportamentos sexuais de estudantes da Escola Superior de Enfermagem de Coimbra (ESEnfC), da Escola Superior de Saúde Dr. Lopes Dias (ESALD), da Universidade Federal de Juíz de Fora (UFJF)-Brasil; analisar a influência dos conhecimentos sobre sexualidade e a adesão aos métodos contraceptivos; analisar a influência de ter namorado(a), do auto-conceito e dos comportamentos nas atitudes sexuais desses estudantes. 


\section{POPULAÇÃO E AMOSTRA}

A População é constituída pelos estudantes de ensino superior (entre os 18 e os 24 anos), matriculados no $1^{0}$ ano das instituições: Escola Superior de Saúde Dr. Lopes Dias (ESALD) do Instituto Politécnico de Castelo Branco, Escola Superior de Enfermagem de Coimbra (ESEnfC) e Universidade Federal de Juíz de Fora (UFJF), Brasil. A amostra é constituída pelos estudantes dessas três instituições.

Para que a amostra apresente um elevado grau de homogeneidade constituem critérios de inclusão:

- ser estudante do ensino superior; estar matriculado no $1^{0}$ ano; ter entre 18 e 24 anos; ser solteiro.

Foram inquiridos 547 estudantes, tendo sido considerados válidos os questionários de 494 respondentes, correspondendo a 258 estudantes da Escola Superior de Enfermagem de Coimbra (ESENFC), 141 estudantes da Escola Superior de Saúde Dr. Lopes Dias (ESALD) e 95 estudantes da Faculdade de Enfermagem da Universidade Federal de Juiz de Fora (UFJF).

\section{METODOLOGIA}

Para a colheita de dados foi utilizado o questionário de auto-preenchimento constituído por um grupo de caracterização dos indivíduos que constituem a amostra e outras variáveis como: estado civil; namoro; experiências sexuais; métodos contraceptivos; religião e informação sobre sexualidade. $E$ uma Escala de Comportamentos e Atitudes (ECA) em relação à sexualidade, um instrumento de avaliação a nível psicossocial e comportamental, poderão aparecer agrupados por dimensões: Intenção comportamental; Reações; Norma subjetiva; Perceção de control. Comportamentos em relação à sexualidade: Intenção comportamental: praticar "sexo seguro", que é entendido como uso de preservativo. Reações: sentimentos positivos e negativos do sujeito Face à utilização do preservativo e crenças sobre as consequências positivas ou negativas associadas ao desempenho desse comportamento. Norma subjetiva: julgamento subjetivo sobre 0 grau de aprovação-desaprovação de certas pessoas significativas para o sujeito (pais, amigos, companheiro) sobre 0 uso de preservativo. Percepção de controlo: expectativas de auto-eficácia, julgamento subjetivo sobre o grau de convicção na dificuldade ou facilidade de usar (ou fazer usar) o preservativo no próximo encontro sexual.

A resposta é medida através de uma escala de Likert que varia entre 1 e 7, com 18 itens, que avaliam a intenção comportamental, reações, normas e percepção do controlo comportamental face ao uso do preservativo no próximo encontro sexual e estratégias de prevenção privilegiadas.

Foram tidos em consideração os procedimentos formais e éticos relativamente à participação de indivíduos em estudos de investigação, nomeadamente esclarecimento dos sujeitos participantes, relativamente aos objectivos da investigação e à garantia do anonimato.

Para a análise estatística, descritiva e inferencial, recorreu-se ao programa estatístico Package Analyses Software (PASW), versão 20.

Foi utilizada estatística descritiva analítica, recorrendo-se às frequências absolutas e relativas, medidas de tendência central-média e medidas de dispersão-desvio padrão.

Utilizaram-se as técnicas não paramétricas para estudar a correlação e a diferença entre médias entre grupos. Para avaliar a correlação entre as variáveis, 0 teste $t$ student, 0 teste de Mann-Whitney e o Kruskall-Wallis (Maroco, 2007; Pereira, 2005).

Para a descrição das características da amostra, em função das variáveis, foram utilizados diferentes procedimentos estatísticos univariados.

Foram ainda utilizados o teste de Qui-Quadrado, o teste de Mann-Whitney e o teste de KruskallWallis e a análise de correlação de Spearman.

0 nível de significância utilizado foi de 0,05. 


\section{COMPORTAMENTOS E ATITUDES dOS ESTUDANTES DO ENSINO SUPERIOR EM RELAÇÃO À SEXUALIDADE}

\section{RESULTADOS}

Dos estudantes da UFJF a média (M) de idades situa-se no 19,93 anos, com um desvio padrão (DP) de 1,50 , sendo $85,3 \%$ do género feminino e $14,7 \%$ do género masculino, os estudantes da ESENFC têm uma média de idades de 18,77 anos e um desvio padrão de 0,97 , são do género feminino $85,3 \%$ e do género masculino $14,7 \%$, os da ESALD apresentam uma média de idades de 18,61 anos e um desvio padrão de 1,02, predominando 0 género feminino com $74,5 \%$ e o masculino com $25,5 \%$.

Quanto à religião a maioria dos estudantes, pertencente a qualquer uma das instituições são católicos, 81,1\%, 87,2\% e 87,9\% respetivamente (UFJF, ESENFC, ESALD). Sobre a prática religiosa, os alunos da UFJF são maioritariamente praticantes $(54,7 \%)$, o que não se verifica na ESENFC em que $74 \%$ não são praticantes e na ESALD 75,9\% também não são praticantes.

Quando inquiridos sobre se têm namorado, 58,9\%, dos estudantes da UFJF têm namorado, enquanto a maioria dos alunos da ESENFC e da ESALD não têm namorado, $(57,8 \%),(63,1 \%)$.

Relativamente à idade com que teve a 1a relação sexual, 29,5\% e 19,4\% dos alunos da UFJF e da ESENFC, iniciaram a sua atividade sexual aos 16 de idade, enquanto $19,1 \%$ dos alunos da ESALD iniciaram aos 17 anos de idade.

Do total dos estudantes, já tiveram relações sexuais, $68,4 \%$ da UFJF, $67,8 \%$ da ESENFC e $56 \%$ da ESALD.

Voltaram a ter relações no último mês com uma frequência de 3 vezes, 10,5\% UFJF, 6,2\% ESENFC e de 4 vezes $6,4 \%$ ESENFC

0 método contracetivo mais utilizado pelos jovens da UFJF, da ESENFC e da ESALD foi o preservativo com $31,6 \%, 33,3 \%$ e $44 \%$ respetivamente.

Do total da amostra (as 3 instituições) analisando a distribuição por idade e género, verifica-se que a maioria se situa-se nos 18 anos $(45,7 \%)$, idade mais frequente no ingresso ao ensino superior, seguindo-se os 19 anos com (30,6\%), correspondendo a frequência mais baixa aos 24 anos. Quanto ao género predomina o feminino, com $82,2 \%$.

A maioria dos estudantes são católicos ( $86,2 \%)$, dos quais $69 \%$ não são praticantes.

Sobre se tem ou não namorado(a) a maioria $(56,1 \%)$ não tem namorada. Dos que têm namorada 18\% namoram há 1 ano, seguindo-se os que namoram há 2 anos (10,7\%).

Já tiveram relações sexuais $64,6 \%$ dos estudantes, tendo a maioria iniciado a atividade sexual aos 16 anos (18,8\%), seguindo-se os 17 e 18 anos com 15,2\% e 12,6\% respetivamente. Tiveram relações sexuais no último mês $36,8 \%$ dos estudantes, correspondendo a maioria a 3 vezes $(5,7 \%)$, seguindo-se 2, 4, 5 e 10 vezes respetivamente $(4,7 \%, 4,7 \%, 4,3 \%$ e $4 \%)$. A maioria dos que namoram responderam que não tiveram relações sexuais com outro parceiro (65\%), durante 0 período de namoro.

Dos que já tiveram relações sexuais existem $36 \%$ que utiliza só preservativo, seguindo-se uma dupla proteção, preservativo, pilula em $26,1 \%$.

Quando questionados sobre a informação sobre sexualidade, a maioria $(53 \%)$ considera "Boa" a informação sobre sexualidade. Os inquiridos percecionam os colegas e amigos, o companheiro habitual e a imprensa e televisão (por ordem decrescente de importância) como agentes importantes na sua formação e informação acerca da sexualidade.

Os resultados obtidos da correlação entre a idade e as atitudes sexuais evidenciaram correlações positivas e estatisticamente significativas $(p<0,05)$. Para as atitudes sexuais em função da utilização de método contraceptivo, existe diferença estatisticamente significativa $(p<0,05)$. 0 que parece indicar que os indivíduos que não se preocupam com o risco de ISTs e gravidez apresentam valores de maior proximidade em relação a este tipo. 
Os resultados obtidos quanto ao facto de ter ou não namorado(a) permitiram verificar diferença estatisticamente significativa em relação às atitudes sexuais $(p<0,05)$.

Já relativamente à informação dos inquiridos sobre sexualidade, verificou-se diferença estatisticamente significativa $(p<0,05)$.

Para a ECA as pontuações mais elevadas situam-se nas respostas dadas às perguntas "Insistirei na utilização do preservativo com um novo(a) ou primeiro(a) companheiro(a) sexual" ( $M=6,84$; $\mathrm{DP}=0,61$ ); "Até que ponto é que as seguintes pessoas consideram que deve ou não utilizar (ou fazer utilizar) o preservativo num futuro encontro sexual: b) os meus pais" ( $M=6,77 ; \mathrm{DP}=0,75)$; "Até que ponto é que as pessoas que são mais importantes e significativas para si, aprovam que utilize (ou faça utilizar) o preservativo no seu futuro encontro sexual" ( $M=6,68$; $D P=0,73$ ); "Em sua opinião, qual o grau de probabilidade de ocorrência dos seguintes resultados, em consequência da utilização do preservativo no seu futuro encontro sexual: c) Protecção contra doenças sexualmente trans-

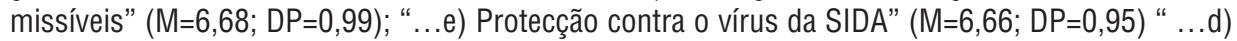
Evitamento e prevenção da gravidez ( $M=6,60$; $D P=0,87)$; "Até que ponto é que as seguintes pessoas consideram que deve ou não utilizar (ou fazer utilizar) o preservativo num futuro encontro sexual: a) os meus amigos" $M=6,51$; $D P=0,96$ ); "Eu tenciono utilizar (ou fazer com que 0 outro utilize) um preservativo no meu futuro encontro sexual" ( $M=6,36$; $D P=1,57)$.

"Gosto sempre de me sair bem das coisas que faço" ( $M=4,33$ e $D P=0,68)$. "De modo geral tenho por hábito enfrentar e resolver os meus problemas" ( $M=4,09 ; \mathrm{DP}=0,74)$. "Considero-me uma pessoa agradável no contacto com os outros" ( $M=4,00$; $D P=0,67)$.

Observam-se diferenças estatisticamente significativas nas pontuações obtidas na subescala de Reações em função do facto de os inquiridos terem tido ou não relações sexuais $(p<0,001)$. Em particular, observa-se que os inquiridos que não tiveram relações sexuais, tendem a pontuar mais alto nesta subescala.

\section{DISCUSSÃO}

Relativamente à diferença de géneros, considerando que no nosso estudo predomina o genero feminino, podemos analisar sobre dois aspetos, um ligado aos aspetos genéticos e hormonais e 0 outro relacionado com fenómenos de natureza cultural. Debruçamo-nos sobre algumas diferenças de atitudes e comportamentos sexuais dos homens e das mulheres, nomeadamente sobre o relacionamento sexual.

Os namoros, são considerados por Frias (2006) como um elemento de grande relevância na adolescência, "contribuindo para o desenvolvimento afectivo-sexual da pessoa" (p. 129). A maioria dos estudantes da nossa amostra referem ter namorada/o, o mesmo autor refere que "as relações amorosas e as práticas sexuais na adolescência podem assumir vários papéis, funcionando como alívio de angústia, meio de obter uma aceitação perante o(a) parceiro(a) ou o grupo, forma de suprir carências afectivas, de auto-afirmação, de manifestação de inconformismo e de rebeldia, ou ainda como uma tentativa de obter um maior grau de dependência" (Frias, 2006, p. 124).

Deste modo, a prática da sexualidade só é considerada como responsável e satisfatória se 0 adolescente possuir competências cognitivas e afetivas para tomar, por si só, decisões sobre a sua intimidade e sobre o que se sente preparado para fazer (Antunes, 2007).

Todavia, perante a ausência de um meio familiar e escolar que promova o desenvolvimento da sexualidade e dos afetos, o que se sucede é a incapacidade de construção da personalidade e de relações afetivas sólidas e, também, a incapacidade de aquisição de valores (que usualmente são divulgados pelos familiares). Perante esta situação, são comuns as relações casuais e os comportamentos de risco, e perante o despertar da sexualidade que ocorre na adolescência, as decisões 


\section{COMPORTAMENTOS E ATITUDES DOS ESTUDANTES DO ENSINO SUPERIOR EM RELAÇÃO À SEXUALIDADE}

tomadas nem sempre têm por base condições de maturidade cognitiva e afectiva ideais (Vaz, 2011). 0 jovem, na adolescência, requer liberdade e capacidade deliberativa própria, para se tornar adulto, independente e responsável por si mesmo, por isso é inevitável que a relação entre pais-filhos sofra alterações no decorrer da mesma.

A população universitária está na faixa etária que mais recorre à interrupção da gravidez, alerta a APF, que concorda com a distribuição de contraceptivos nas instituições do ensino superior. Num estudo realizado por Vilar (2008) sobre a interrupção da gravidez, conclui-se que os dois grupos que mais recorrem a esse método são os jovens, por volta dos 20 e os adultos por volta dos 30 anos, estamos portanto exactamente a falar do grupo da população universitária objecto do nosso estudo como um grupo prioritário a nível da prevenção em termos de saúde sexual.

\section{CONCLUSÃO}

Se por um lado se tem verificado uma maior preocupação para formar/informar os jovens na área da sexualidade e da contraceção, através das escolas, dos pais e das informações disponiveis, também é verdade que a entrada no ensino superior leva à existência de comportamentos e atitudes pouco reflectidas, nem sempre com resultados agradáveis.

0 enfermeiro deve proporcionar um ambiente calmo e seguro para a realização do diálogo com o jovem, facultando informação adequada e concisa, escutando as preocupações e respeitando 0 sigilo profissional e a confidencialidade de toda a informação, decisões e ações, pois o jovem, possui 0 direito de ter a sua opinião, de a expressar e de procurar ajuda e orientação. Pretendendo-se assim que 0 enfermeiro desempenhe 0 seu papel de educação para a sexualidade tanto nas instituições de saúde (hospitais e centros de saúde) como nas instituições de ensino.

\section{BIBLIOGRAFIA}

Antunes, M. (2007). Atitudes e Comportamentos sexuais de estudantes do ensino superior. Coimbra: Formasau.

Coleman, L. M., \& Testa, A. (2008). Sexual health knowledge, attitudes and behaviours: variations among a religiously diverse sample of young people in London, UK. Ethnicity \& Health, 13, 5572.

Díaz, M., Mello, M. B. d., Sousa, M. H. d., Cabral, F., Silva, R. d. C. e., Campos, M., \& Faúndes, A. (2005). Outcomes of three different models for sex education and citizenship programs concerning knowledge, attitudes, and behavior of Brazilian adolescents. Cadernos de Saúde Publica, 21, 589-597.

Frias, A. (2006). Expressões da sexualidade e atitudes contracetivas dos adolescentes.

Revista Servir, Maio - Junho. 54 (3), 121-130.

Frias, A. \& Chora, A. (2013). Adolescents Sexual Practices. Atención Primaria. Vol 45 (mayo), 82. España: Sociedad Española de Medicina de familia y Comunitaria. ISSN:0212-6567

Gouveia, P., Leal, I., Maroco, J., \& Cardoso, J. (2010). EAS- Versão adolescentes (EAS-A). In I. Leal \& J. Maroco (Eds.), Avaliação em sexualidade e parentalidade (pp. 59-74). Porto: Legis Editora.

Jesus, A. A. (2011). Educação Sexual: Uma metodologia formal vs lúdica/emocional. Mestrado, Universidade de Lisboa, Lisboa.

López, F. (2009). La educación sexual. Madrid: Biblioteca Nueva

Ramos, V., Carvalho, C. \& Leal, I. (2005). Atitudes e Comportamentos Sexuais de

Mulheres Universitárias: A Hipótese do Duplo Padrão Sexual. Análise Psicológica 23(2):173186.

Robin, L., Dittus, P., Whitaker, D., Crosby, R., Ethier, K., Mezoff, J., . . Pappas-Deluca, K. (2004). Behavioral interventions to reduce incidence of HIV, STD, and pregnancy among adolescents: a 
decade in review. Journal of Adolescent Health, 34, 3-26.

Vaz; A. (2011) Atitudes e comportamentos dos adolescents face à sexualidade. Tese de doutoramento: Universidade da Extremadura.

Vilelas, J. (2009). A influência da família e da escola na sexualidade do adolescente. Coimbra: Formasau. 195 p. ISBN 978-989-8269-02-7. 
